\title{
A humanização do cuidado em saúde no contexto da formação do enfermeiro em um hospital de ensino
}

\author{
The humanization of health care in the context of the training of nurses in a teaching hospital
}

La humanización del cuidado de la salud en el contexto de la formación de enfermeras en un hospital docente

Recebido: 19/02/2021 | Revisado: 28/02/2021 | Aceito: 07/03/2021 | Publicado: 15/03/2021

Nadja da Fonseca Veloso
ORCID: https://orcid.org/0000-0001-5985-5300
Universidade do Estado do Pará, Brasil
E-mail: nadveloso@ hotmail.com
Daniele Lima dos Anjos Reis
ORCID: https://orcid.org/0000-0002-8447-6828
Universidade do Estado do Pará, Brasil
E-mail: anjo.daniele@ @otmail.com
Taiana Moita Koury Alves
ORCID: https://orcid.org/0000-0001-8263-9315
Universidade Federal do Pará
E-mail: taikoury@yahoo.com.br
Rosângela Lima da Silva
Universidade do Estado do Pará
E-mail: rosangela.silva@ uepa.br
ORCID: https://orcid.org/000-0003-2000-4343
Bruna Karine Oliveira do Carmo
ORCID: https://orcid.org/0000-0002-1220-9691
Universidade Federal do Pará
E-mail: brunakarine_bk@ @otmail.com
Antônio Breno Maia de Araújo
ORCID: https://orcid.org/0000-0002-1868-5103
Universidade Federal do Pará
E-mail: antoniobrenomaia@gmail.com

Adele Melo Silva

ORCID: https://orcid.org/0000-0003-2979-7474

Empresa Brasileira de Serviços Hospitalares-EBSERH, Brasil

E-mail: adele.admhosp.hu@gmail.com

Ely dos Santos Farias

ORCID: https://orcid.org/0000-0002-1386-2000

Empresa Brasileira de Serviços Hospitalares-EBSERH, Brasil

effarias13@hotmail.com

Ana Caroline Guedes Souza Martins

ORCID: https://orcid.org/0000-0001-7185-8520

Universidade do Estado do Pará, Brasil

E-mail: carolguedes.devs@hotmail.com

Marllon Rodrigo Sousa Santos

ORCID: https://orcid.org/0000-0002-8447-6828

Universidade Federal do Pará, Brasil

E-mail: marlon-rodrigo@ hotmail.com

Angélica Menezes Bessa Oliveira

ORCID: https://orcid.org/0000-0001-7644-4538 Universidade do Estado do Pará, Brasil

E-mail: angelbssa@hotmail.com

Renato da Costa Teixeira

ORCID: https://orcid.org/0000-0002-4073-205X Universidade do Estado do Pará, Brasil

E-mail: renatocteixeira@uepa.br

\section{Resumo}

Este estudo tem como objetivo conhecer a percepção dos enfermeiros preceptores quanto à humanização do cuidado, no contexto da formação do enfermeiro, em um hospital de ensino. Trata-se de uma pesquisa descritiva, com abordagem quanti-qualitativa, realizada por meio da aplicação de um formulário on-line cuja amostra foi composta por 17 enfermeiros preceptores que atuam em unidades de internação. Quanto ao perfil, são do sexo feminino 
(76,5\%), de 31 a 40 anos (82,3\%), atuam de 0 a 1 ano no setor atual, 5 a 10 anos de formação (82,3\%), especialização lato sensu $(94,1 \%)$, cursou residência $(35,2 \%)$ e cursou mestrado $(23,5 \%)$, possui experiência em pesquisa $(52,9 \%)$, tem experiência com docência tradicional nível técnico (47,0\%), não possui experiência com metodologias ativas (47,0\%), não possui experiência com Educação à Distância $(41,1 \%)$, tem experiência com preceptoria $(52,9 \%)$ e foram preceptores em 2020 (64,7\%). 70,6\% alegam conhecer o termo "Humanização do Cuidado" e descreveram este conceito como entendimento de forma integral do ser humano (100\%), empatia (100\%) e respeito (100\%), tratamento humano $(88,2 \%)$ e ambiência $(76,5 \%)$. Afirmam que a humanização do cuidado ao paciente no contexto da formação do enfermeiro se traduz em aprendizagem a ser desenvolvida pelo aluno no contato permanente e reflexivo com as situações reais de trabalho cotidiano $(82,4 \%)$ e deram nota 4 para sua prática quanto à humanização $(70,6 \%)$, de um total de 5 pontos. Acredita-se que essa pesquisa seja relevante como forma de analisar como a preceptoria influencia na construção do processo de humanização do residente/estudante de enfermagem.

Palavras-chave: Ensino; Preceptoria; Enfermagem; Humanização.

\begin{abstract}
This study aims to understand the perception of nurse preceptors regarding the humanization of care, in the context of the training of nurses, in a teaching hospital. It is a descriptive research, with a quanti-qualitative approach, carried out through the application of an online form whose sample was composed of 17 nurse tutors who work in inpatient units. As for the profile, they are female $(76.5 \%), 31$ to 40 years old $(82.3 \%)$, they work from 0 to 1 year in the current sector, 5 to 10 years of training $(82.3 \%)$, lato sensu specialization $(94.1 \%)$, attended residency $(35.2 \%)$ and studied master's degree $(23.5 \%)$, has research experience $(52.9 \%)$, has experience with traditional teaching at technical level $(47.0 \%)$, have no experience with active methodologies $(47.0 \%)$, have no experience with Distance Education $(41.1 \%)$, have experience with tutoring (52.9\%) and were tutors in $2020(64.7 \%) .70 .6 \%$ claim to know the term "Humanization of Care" and described this concept as an integral understanding of the human being (100\%), empathy $(100 \%)$ and respect $(100 \%)$, human treatment $(88.2 \%)$ and ambience $(76.5 \%)$. They affirm that the humanization of patient care in the context of the training of nurses translates into learning to be developed by the student in permanent and reflective contact with the real situations of daily work $(82.4 \%)$ and rated 4 for their practice regarding the humanization $(70.6 \%)$, out of a total of 5 points. It is believed that this research is relevant as a way of analyzing how preceptorship influences the construction of the nursing student / student humanization process.
\end{abstract}

Keywords: Teaching; Preceptorship; Nursing; Humanization.

\title{
Resumen
}

Este estudio tiene como objetivo comprender la percepción de las enfermeras preceptoras sobre la humanización del cuidado, en el contexto de la formación de enfermeras, en un hospital docente. Se trata de una investigación descriptiva, con abordaje cuanti-cualitativo, realizada mediante la aplicación de un formulario online cuya muestra estuvo compuesta por 17 enfermeras tutoras que laboran en unidades de internación. En cuanto al perfil, son mujeres (76,5\%), 31 a 40 años (82,3\%), laboran de 0 a 1 año en el sector actual, 5 a 10 años de formación (82,3\%), especialización lato sensu $(94,1 \%)$, cursó residencia $(35,2 \%)$ y estudió maestría $(23,5 \%)$, tiene experiencia investigadora $(52,9 \%)$, tiene experiencia con la enseñanza tradicional a nivel técnico $(47,0 \%)$, no tiene experiencia con metodologías activas $(47,0 \%)$, no tienen experiencia en Educación a Distancia $(41,1 \%)$, tienen experiencia en tutorías $(52,9 \%)$ y fueron tutores en 2020 (64,7\%). El 70,6\% afirma conocer el término "Humanización del Cuidado" y califica este concepto como comprensión integral del ser humano (100\%), empatía (100\%) y respeto (100\%), trato humano $(88,2 \%)$ y ambientación $(76,5 \%)$. Afirman que la humanización de la atención al paciente en el contexto de la formación de enfermeras se traduce en aprendizajes a desarrollar por el estudiante en contacto permanente y reflexivo con las situaciones reales del trabajo diario $(82,4 \%)$ y puntuado 4 por su práctica en cuanto a la humanización. $(70,6 \%)$, de un total de 5 puntos. Se cree que esta investigación es relevante como una forma de analizar cómo la tutela influye en la construcción del proceso de humanización del residente / estudiante de enfermería.

Palabras clave: Enseñando; Tutela; Enfermería; Humanización.

\section{Introdução}

No Brasil, um dos desafios para fortalecer o SUS, têm sido as mudanças dos modelos de atenção à saúde, que inclui a transformação nos modos de produzir gestão e cuidado. Como uma das estratégias para a reformulação das práticas assistenciais em saúde, foi criada em 2003 pelo Ministério da Saúde, a Política Nacional de Humanização (PNH) ou HumanizaSUS, uma política pública integrante do Sistema Único de Saúde (SUS) (Freitas \& Ferreira, 2016).

A PNH foi pensada devido às dificuldades para prover um tratamento mais humanizado, especialmente no que se refere à gestão participativa e ao trabalho em equipe, uma vez que promove um comprometimento das práticas de saúde e da responsabilização com os usuários em suas diferentes necessidades, com forte desrespeito aos seus direitos. Fenômenos 
genericamente apontados como desumanos denotam mais que falhas éticas de trabalhadores ou gestores, correspondem a fatos cuja origem revela e expressa determinadas concepções de trabalho e de suas formas de organização (Luis, Caregnato \& Costa, 2017).

É notável a importância das tecnologias de relação, de acesso, acolhimento, produção de vínculo, de encontros de subjetividades, levando a autonomização, as chamadas tecnologias leves para o cuidado em enfermagem (Luis, Caregnato \& Costa, 2017).

Um dos desafios para manter e qualificar a PNH como uma política pública do SUS tem sido a mudança da formação dos profissionais de saúde. No que se refere à enfermagem, as Diretrizes Curriculares Nacionais (DCN) dos cursos de graduação propõem que a formação do enfermeiro deve atender às necessidades sociais da saúde, com ênfase no SUS e assegurar a integralidade da atenção, a qualidade e humanização do atendimento, cujo perfil deve se apresentar como: enfermeiro, com formação generalista, humanista, crítica e reflexiva (Bresolin et al., 2019).

Portanto, as instituições de saúde devem estimular o cuidado humanizado como fator indispensável no cotidiano de quem cuida, além de promover programas institucionais sobre o tema e discussões, tendo em vista o esclarecimento e sistematização dessas ações, além da promoção de debates e trocas de experiências sobre os processos que regem o cotidiano de todos envolvidos, no sentido de integrar a teoria e a prática (Sanches et al., 2016).

Assim, nos espaços de assistência à saúde, muitos profissionais desenvolvem atividades assistenciais e, concomitantemente, atuam como formadores de profissionais de saúde. A essa ação ou prática educativa e formativa, dá-se o nome de preceptoria. Dentre esses profissionais estão os enfermeiros, que alçados à condição de educadores, tornam-se preceptores (Oliveira \& Daher, 2016).

Atuando como um facilitador nesse processo de formação de profissionais com práticas de saúde humanizadas, o preceptor deve apresentar conhecimento teórico, didático e político. Para isto, deve, também, ser e estar preparado para oferecer ao discente a compreensão dos propósitos da enfermagem. Sua experiência e discernimento são fundamentais para interligar a graduação e o mercado de trabalho. Os serviços de saúde constituem cenário desejado pelas Instituições de Ensino Superior (IES) para, tanto quanto à questão da prática e das habilidades específicas, como no que concerne à humanização e ética (Rodrigues et al., 2014).

Nos estudos de Freitas e Ferreira (2016), a figura do professor no ambiente de prática surge como um elemento que facilita o aprendizado da humanização, principalmente por meio da metodologia do ensino pelo exemplo, pois, o discente observa como o professor se relaciona com os usuários nos serviços de saúde e percebe que é possível colocar em prática o que se aprende na teoria.

A formação em saúde deve implicar ações e trocas coletivas, tendo como base práticas concretas de intervenção para que possa ser capaz de gerar novas práticas, por isso, o educando, desde a sua formação acadêmica precisa ser instigado a se instrumentalizar moralmente na vivência da realidade profissional, a fim de tornar-se fortalecido para resolver conflitos cotidianos em seu fazer profissional de maneira ética (Avila et al., 2018).

Oportunizar ao discente a possibilidade de vincular o conhecimento técnico-científico, a competência e a destreza técnica, à habilidade para promover relações interpessoais favoráveis entre si, com docentes, usuários, familiares e demais trabalhadores da saúde é de fundamental importância. Pois, segundo Avila et al., (2018), o próprio educando deve participar e vivenciar os problemas éticos e morais, pois não basta receber orientações passivamente, ele tem de constituir-se como sujeito ativo no processo de educação moral e de construção de si.

Considerando a importância da humanização no cuidado em enfermagem, na perspectiva da PNH, esta pesquisa justifica-se por discutir questões que fundamentam e colaboram para a formação de profissionais com perfil adequado às necessidades e às políticas de saúde do país e com elevada qualificação técnica e científica, cuja atuação esteja pautada pelo 
espírito crítico, de cidadania e compromisso social.

Deste modo, se constitui de relevância social para a área da saúde, podendo até proporcionar novos enfoques diante desta temática, pois a pesquisa tem por objetivo conhecer a percepção dos enfermeiros preceptores quanto à humanização do cuidado, no contexto da formação do enfermeiro, em um hospital de ensino.

\section{Metodologia}

Esta pesquisa caracteriza-se como de caráter exploratória, analítico-descritiva, com abordagem quanti-qualitativa, apoiada na Política Nacional de Humanização. Para Bonin (2018, p. 17), a pesquisa exploratória:

ela se realiza por meio de aproximações empíricas do fenômeno concreto a ser investigado, com o intuito de perceber seus contornos, nuances, singularidades e inclui tatear o fenômeno, explorar aspectos que interessam à problemática em construção, na sua feição concreta.

Para Fleury (2017), a pesquisa descritiva objetiva compreender o fenômeno e descrever a sua distribuição em uma determinada população. Exige do investigador uma série de informações sobre o que deseja pesquisar. Entende-se que a pesquisa descritiva é abrangente, permite analisar o problema em relação aos aspectos sociais, econômicos, políticos e percepções de diferentes grupos, comunidade, e também é utilizada para compreensão de diferentes comportamentos.

A pesquisa qualitativa ocorre no ambiente natural com coleta direta de dados e o pesquisador é o principal instrumento. Os dados coletados são preferencialmente descritivos, em que a preocupação do processo é predominante em relação à do produto. O "significado" que as pessoas dão as coisas e a sua vida são focos de atenção para o pesquisador e, a análise de dados e informações tende a seguir um processo indutivo (Pereira et al., 2018).

Enquanto que a pesquisa quantitativa é aquela em que se coletam e analisam dados quantitativos sobre variáveis. Dessa forma, este tipo de pesquisa é capaz de identificar a natureza profunda das realidades, seu sistema de relações, sua estrutura dinâmica. Ela também pode determinar a força de associação ou correlação entre variáveis, a generalização e objetivação dos resultados através de uma mostra que faz inferência a uma população. Além do estudo da associação ou correlação, a pesquisa quantitativa também pode, ao seu tempo, fazer inferências causais que explicam por que as coisas acontecem ou não de uma forma determinada (Esperón, 2017).

A pesquisa ocorreu em hospital público universitário localizado em Belém, Pará, caracterizado como uma unidade de assistência, ensino e pesquisa, referência regional em pneumologia, infectologia e endocrinologia e diabetes, e referência nacional em Síndrome da Imunodeficiência Adquirida (SIDA).

Participaram da pesquisa 17 enfermeiros preceptores que atuam nos turnos manhã e tarde, períodos de desenvolvimento de preceptorias nas unidades de internação de pacientes.

Os critérios de inclusão foram: enfermeiros de ambos os sexos, que trabalham em unidades de internação de pacientes e atuaram em preceptoria com alunos graduandos e/ou residentes, nos últimos dois anos. Já como critérios de exclusão: profissionais que estejam gozando de férias, licença saúde ou qualquer outro tipo de afastamento. Do total de 35 enfermeiros, 08 estavam afastados por atestado ou férias e 10 se recusaram a participar deste estudo.

A coleta de dados da ocorreu em fevereiro de 2021, por meio da aplicação de um formulário on-line pela plataforma Google Forms composto por questões abertas e fechadas, visando conhecer o perfil dos profissionais e verificar os conhecimentos acerca do tema abordado.

O Google Forms é um método de coleta de dados que garante o anonimato do respondente, porém para que ele seja preenchido é necessário o aceite em participar através da leitura do Termo de Consentimento Livre e Esclarecido (TCLE) e opção "Sim". A qualquer momento pode ser observado como está o andamento da pesquisa, ou seja, a análise pode ser 
realizada em tempo real. Com as respostas qualitativas, é possível entender de maneira mais ampla as percepções dos pesquisados no que se refere aos aspectos perguntados (Monteiro \& Santos, 2019).

Inicialmente foi enviado uma solicitação para a Divisão de Enfermagem do local do estudo, informando sobre a necessidade de disponibilização do link do formulário aos participantes do estudo através de rede social. Em seguida foram solicitados os contatos telefônicos dos profissionais.

Cada participante recebeu um link composto por uma pequena explicação sobre a pesquisa, e acesso direto ao questionário. Foi enviado junto ao link, o Termo de Consentimento Livre e Esclarecido, e após aceitarem, responderam ao questionário. Cada participante tinha livre escolha para acessar o link e responder ou não o questionário. A responsabilidade e risco pelo não recebimento dos questionários respondidos foi unicamente do pesquisador.

O instrumento de coleta de dados foi dividido em duas partes: a primeira sobre a caracterização do perfil dos preceptores; e a segunda, são perguntas fechadas escalonadas organizadas em escala, do tipo escala likert, e uma pergunta aberta, a fim de explorar os sentidos, práticas e vivências dos preceptores perante o tema da humanização do cuidado em saúde.

A escala likert é considerada a escala de verificação mais utilizada em pesquisas que envolvam a opinião de pessoas. Utiliza o escalonamento das opções de resposta, que enfatiza o uso de escalas com cinco alternativas. É, porém, conhecido o fato de muitos respondentes sentirem insegurança para tomar uma posição, e quando se quer evitar o "em cima do muro", recomenda-se o uso de quatro alternativas, evitando o "ponto neutro" (Santino, 2018).

A pesquisa teve aprovação pelo Comitê de Ética e Pesquisa do HUJBB, local onde foi realizado a coleta de dados, sob parecer n. 05777119.0.0000.0017 e atendeu aos preceitos éticos da Resolução nº 466/2012 do Conselho Nacional de Saúde.

A análise estatística foi feita de maneira descritiva e inferencial. Os dados foram analisados e organizados em gráficos e tabelas.

\section{Resultados e Discussão}

A partir dos objetivos propostos os resultados foram organizados em 3 unidades, a saber: Caracterização do perfil dos preceptores; Sentidos, práticas e vivências dos preceptores perante o tema da humanização do cuidado em saúde; E assistência humanizada ao paciente no contexto da formação do enfermeiro.

\subsection{Caracterização do perfil dos preceptores}

O perfil dos participantes do estudo encontra-se na tabela 1, conforme exposto a seguir: 
Tabela 1: Perfil dos participantes do estudo. Belém, Pará, Brasil, 2021.

\begin{tabular}{|c|c|c|c|}
\hline & & $\mathbf{n}$ & $\%$ \\
\hline \multirow[t]{2}{*}{ Sexo } & Feminino & 13 & 76,5 \\
\hline & Masculino & 4 & 23,5 \\
\hline \multirow[t]{3}{*}{ Idade } & Menor que 30 anos & 1 & 5,8 \\
\hline & 31 a 40 anos & 14 & 82,3 \\
\hline & Maior que 40 amos & 2 & 11,7 \\
\hline \multirow[t]{3}{*}{ Tempo de formação (graduação) } & 5 a 10 anos & 14 & 82,3 \\
\hline & 11 a 20 anos & 2 & 11,7 \\
\hline & Acima de 20 anos & 1 & 5,8 \\
\hline \multirow[t]{10}{*}{ Setor de atuação } & Clínica Cirúrgica & 2 & 11,7 \\
\hline & Clínica Médica & 3 & 17,6 \\
\hline & UDIP & 3 & 17,6 \\
\hline & Pneumologia & 1 & 5,8 \\
\hline & UCISI & 2 & 11,7 \\
\hline & USR & 1 & 5,8 \\
\hline & UCM & 1 & 5,8 \\
\hline & UTI & 2 & 11,7 \\
\hline & Pediatria & 1 & 5,8 \\
\hline & UASCA & 1 & 5,8 \\
\hline \multirow[t]{3}{*}{ Tempo de atividade no setor } & 0 a 1 ano & 6 & 35,2 \\
\hline & 2 anos & 7 & 41,1 \\
\hline & 3 anos & 4 & 23,5 \\
\hline \multirow[t]{4}{*}{ Pós-graduação } & Lato sensu & 16 & 94,1 \\
\hline & Residência & 6 & 35,2 \\
\hline & Mestrado & 4 & 23,5 \\
\hline & Doutorado & 0 & 0,0 \\
\hline
\end{tabular}

Fonte: Roteiro de entrevista aplicado pela autora (2021).

De acordo com os dados da Tabela 1, houve predominância do sexo feminino (76,5\%), em que 82,3\% estão na faixa etária de 31 a 40 anos e atuam nos setores: Clínica Cirúrgica, Clínica Médica, UDIP, Pneumologia, UCISI, USR, UCM, UTI, UASCA, Pediatria. 35,2\% atuam de 0 a 1 ano no setor atual. Quanto ao tempo de formação, 82,3\% tem de 5 a 10 anos, $94,1 \%$ possui especialização lato sensu, 35,2\% cursou residência e 23,5\% cursou mestrado.

Estes achados corroboram com o estudo de Costa (2017), no qual 64\% dos participantes estão na faixa etária entre 31 a 40 anos, o que implica no tempo maior de formação profissional, consequentemente, com maior conhecimento prático devido ao maior tempo no campo de formação. Em menor proporção, apresenta-se o quantitativo de profissionais com idade abaixo dos 30 anos, cerca de $29 \%$ do total de participantes.

Em relação ao perfil esperado pelos profissionais preceptores, acredita-se que o maior tempo de atuação como agente formador facilitará no desenvolvimento prático dos residentes de enfermagem e maior credibilidade no processo de ensino e aprendizagem no campo prático (Costa, 2017).

Quanto ao perfil de tempo de formação profissional dos preceptores de enfermagem, destaca-se que 65\% destes são graduados há pelo menos 5 anos e outros há 10 anos, o que implica que os profissionais têm um tempo maior de atuação na prática. Cerca de 14\% do grupo de preceptores são graduados entre 1 e 5 anos (Costa, 2017).

Nesse aspecto, relacionar o tempo de formação com o tempo de prática como preceptor facilitará a abordagem prática em campo com os residentes, implicando em maior produção de conhecimento prático e científico, e desenvolvimento de habilidades e competências. Na prática, o desenvolvimento de habilidade e competências no ambiente de aprendizagem será facilitado a partir de conhecimentos prévios e experiências dos profissionais preceptores, que desenvolverão junto aos residentes uma aprendizagem significativa (Costa, 2017). 
Nesse mesmo discurso, acredita-se que os preceptores de enfermagem sejam profissionais habilitados no campo de atuação prático, com técnicas e conhecimento científico em suas especialidades, tendo em vista que esta é uma exigência da legislação da preceptoria em enfermagem.

Com relação a distribuição dos participantes quanto a experiência em pesquisa, tempo de atuação na preceptoria e experiência em educação dos preceptores, os dados estão apresentados na Tabela 2 e Gráfico 1, a seguir.

Tabela 2: Distribuição dos participantes quanto a experiência em pesquisa e tempo de atuação na preceptoria. Belém, Pará, Brasil, 2021.

\begin{tabular}{cccc}
\hline & & $\mathbf{n}$ & $\mathbf{\%}$ \\
\hline Experiência em pesquisa & Sim & 9 & 52,9 \\
& Não & 8 & 47,0 \\
Ano em que atuou na preceptoria & Nunca atuou & 2 & 11,7 \\
& 2020 & 11 & 64,7 \\
& 2021 & 4 & 23,5 \\
\hline
\end{tabular}

Fonte: Roteiro de entrevista aplicado pela autora (2021).

Gráfico 1: Experiência em educação dos preceptores. Belém, Pará, Brasil, 2021.

\section{Experiência em Educaçāo}

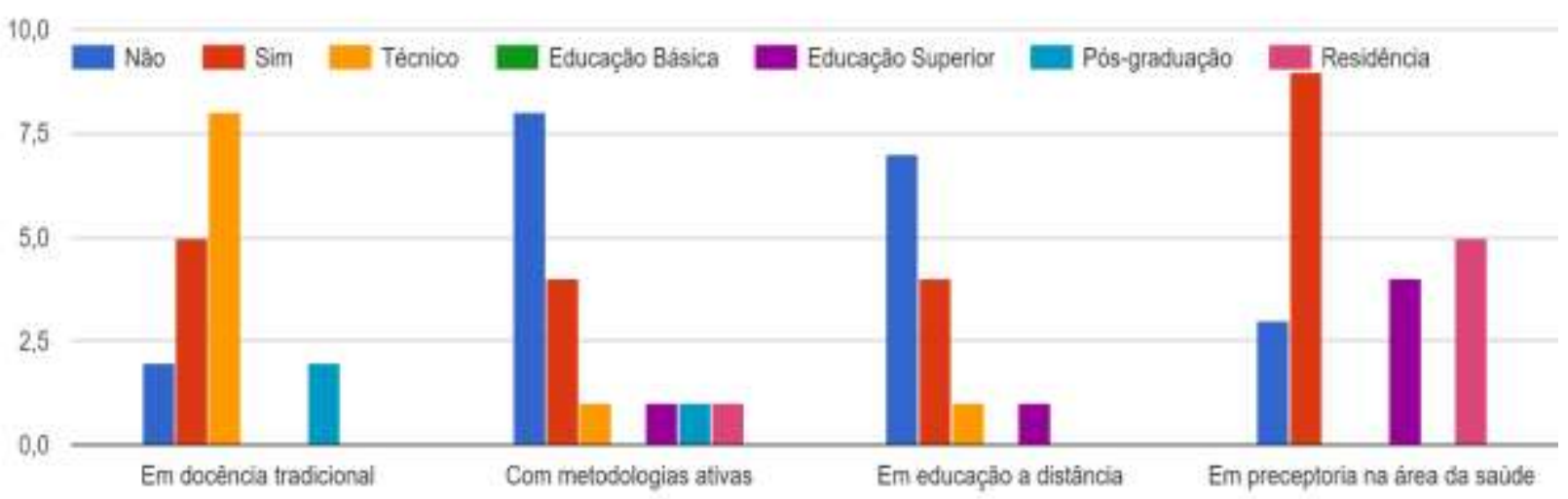

Fonte: Roteiro de entrevista aplicado pela autora (2021).

Quanto à experiência em pesquisa, 52,9\% afirmou que sim, 47,0\% afirma ter experiência com docência tradicional no ensino nível técnico, enquanto que este mesmo percentual alega nunca ter tido experiência com metodologias ativas. Constatou-se que $41,1 \%$ alega não possuir experiência com Educação à Distância, 52,9\% alega ter experiência com preceptoria e 64,7\% dos entrevistados atuaram como preceptores no ano de 2020.

Estes achados corroboram com o estudo de Costa (2017), quanto ao tempo de atuação como preceptor de enfermagem, em que a maioria atua na preceptoria há um período entre 1 e 3 anos $(71,4 \%)$. Nesse mesmo questionamento, percebe-se que que esse tempo de atuação enquanto preceptor é mediano, tendo em vista o tempo de formação como profissionais de enfermagem, porém, em sua maioria, estes preceptores são ex-residentes deste mesmo programa de residência de enfermagem. Por serem exresidentes, acredita-se que conhecem o programa e a filosofia deste, isso facilita a abordagem prática e científica destes residentes em campo prático. 


\subsection{Sentidos, práticas e vivências dos preceptores perante o tema da humanização do cuidado em saúde}

Dentro dessa temática, observou-se os conhecimentos dos participantes da pesquisa acerca do termo "humanização do cuidado em saúde", e o conceito de humanização do cuidado ao paciente, conforme expresso no Gráfico 2 e Tabela 3.

Gráfico 2: Conhecimento dos preceptores sobre o termo "Humanização do cuidado em saúde”. Belém, Pará, Brasil, 2021.

\section{Você conhece o termo "Humanização do Cuidado"? \\ 17 respostas}
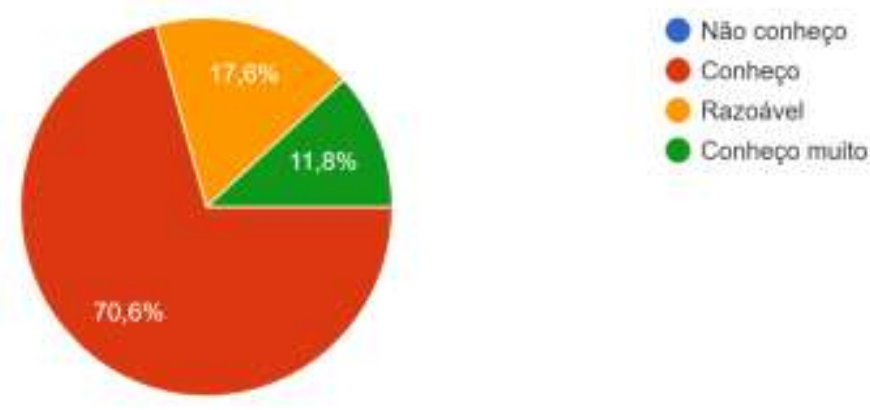

Fonte: Roteiro de entrevista aplicado pela autora (2021).

Tabela 3: Conceito de humanização do cuidado ao paciente pelos enfermeiros preceptores. Belém, Pará, Brasil, 2021.

\begin{tabular}{lll}
\hline \multicolumn{1}{c}{ Conceitos De Humanização } & N & $\%$ \\
\hline Carinho & 4 & 23,5 \\
\hline Filas desnecessárias & 4 & 23,5 \\
\hline Entendimento de forma integral do ser humano & 17 & 100 \\
\hline Descaso e descuido com as pessoas & 0 & 0 \\
\hline Empatia & 17 & 100 \\
\hline Incapacidade de lidar com histórias de vida, sempre singulares e complexas & 0 & 0 \\
\hline Tratamento humano & 15 & 88,2 \\
\hline Discriminação & 1 & 5,9 \\
\hline Respeito & 17 & 100 \\
\hline Intimidação e submissão a práticas e procedimentos desnecessários & 0 & 0 \\
\hline Ambiência & 13 & 76,5 \\
\hline Exclusão e abandono & 0 & 0 \\
\hline Manutenção dos materiais para a oferta de um cuidado integral & 12 & 70,6 \\
\hline Ausência de interação entre o sujeito que recebe o cuidado e o profissional & 0 & 0 \\
\hline Cuidado centrado no humano & 12 & 70,6 \\
\hline Comentários inoportunos e a utilização de rótulos e apelidos para se referir ao & 0 & 0 \\
paciente & 0 & 0 \\
\hline Barulhos constantes & 0 & 0 \\
\hline Falta de privacidade do paciente & 15 \\
\hline
\end{tabular}

Fonte: Roteiro de entrevista aplicado pela autora (2021). 
Com relação ao tema humanização, quando indagados se conhecem o termo "Humanização do Cuidado", 70,6\% alegou que sim. Descreveram o conceito de humanização do cuidado ao paciente, através das seguintes expressões: "entendimento de forma integral do ser humano" (100\%), "empatia" (100\%), "respeito" (100\%), "tratamento humano" (88,2\%), "ambiência" (76,5\%), "manutenção dos materiais para a oferta de um cuidado integral" (70,6\%) e "cuidado centrado no humano" (70,6\%).

\subsection{Assistência humanizada ao paciente no contexto da formação do enfermeiro}

Nos Gráficos 3 e 4 é possível visualizar a opinião dos preceptores acerca da humanização do cuidado ao paciente no contexto da formação do enfermeiro e a nota que os preceptores dariam para sua prática, na busca de uma assistência humanizada aos pacientes.

Gráfico 3: A humanização do cuidado ao paciente no contexto da formação do enfermeiro. Belém, Pará, Brasil, 2021.



Fonte: Roteiro de entrevista aplicado pela autora (2021). 
Gráfico 4: Nota sobre a prática na busca da assistência humanizada. Belém, Pará, Brasil, 2021.

\section{Diante dos desafios impostos pela prática, na condição de preceptor, que nota você daria para a sua prática, na busca de uma assistência humanizada ao paciente. 17 respostas}

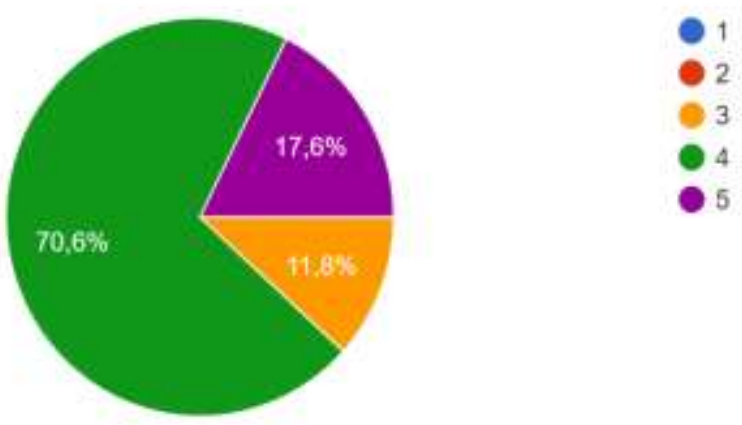

Fonte: Roteiro de entrevista aplicado pela autora (2021).

Sobre como consideram a humanização do cuidado ao paciente no contexto da formação do enfermeiro, $82,4 \%$ afirmam que o valor se traduz em aprendizagem a ser desenvolvida pelo aluno no contato permanente e reflexivo com as situações reais de trabalho cotidiano.

Ao serem questionados que nota dariam para a sua prática, na busca de uma assistência humanizada ao paciente, 70,6\% deram nota 4 , de um total de 5 pontos.

Observou-se que os enfermeiros preceptores, em sua maioria, elencaram uma nota considerada boa para seu desempenho enquanto a assistência humanizada. Acredita-se que por já terem um tempo de formação mínimo de 5 anos, tenham acumulado experiências sobre o tema ao longo dos anos e pela maioria já ter tido experiência com a preceptoria.

Estes achados corroboram com o estudo de Chernicharo et al., (2014) que afirma que a humanização na assistência é caracterizada pelos profissionais de enfermagem como uma prática de todos os profissionais, por estar embasada em uma relação profissional/cliente, por incluir características pessoais, por olhar para as necessidades, pelo diálogo, escuta atentiva, visão holística, empatia, valores morais e éticos, e por incluir questões subjetivas como o amor, o pensamento, a valorização do ser, estabelecimento de vínculo, atenção, o querer, compreensão e carinho. Em contrapartida, os profissionais caracterizam a não humanização como um ato mecânico, tratando a doença, e não o ser humano, com falta de comunicação, agindo somente pela técnica, a carga horária de trabalho exaustiva, problemas institucionais e problemas relacionados aos recursos materiais, colocando a tecnologia em evidência.

Portanto, espera-se de um enfermeiro execute elementos básicos em suas atividades cotidianas, como por exemplo, que este seja acolhedor, pois é a partir do tratamento respeitoso e saudoso que se iniciam e estreitam laços importantes em situações estressoras da vida nas quais mesmo um simples local de espera pode tornar-se acolhedor (Fonseca et al., 2020).

O estudo de Shimazu (2018) descreve o desenvolvimento de uma escala de aprendizagem experimental do preceptor, baseada na teoria da aprendizagem experiencial de Kolb, que valoriza a experiência pessoal no aprendizado. O instrumento é um questionário de auto avaliação curto e fácil de usar, podendo ser empregado em programas de treinamento de preceptores. Outra estratégia de desenvolvimento individual são os feedbacks acerca de sua atuação como preceptores, e também dos coordenadores do programa de residência, bem como dos alunos. 
O conteúdo para formação de preceptores deve basear-se nas necessidades de aprendizagem do participante e no seu estilo de aprendizado, utilizando métodos instrucional ativos em um ambiente seguro e solidário (Cox et al., 2017).

$\mathrm{O}$ apoio para que o preceptor vivencie processos formativos é algo necessário e importante para que ele tenha a possibilidade de refletir criticamente sobre os processos de trabalho, ampliando assim o olhar para as questões sobre humanização em saúde (Fonseca et al., 2020).

As práticas relacionadas ao desenvolvimento de habilidades interdisciplinares são consideradas um mecanismo essencial para atendimento de alta qualidade e segurança dentro dos serviços de saúde, propiciando o desenvolvimento do trabalho coletivo pautado na integralidade das ações de saúde (Fonseca et al., 2020).

É genuíno o interesse dos preceptores na promoção da aprendizagem que não seja apenas do seu núcleo de ensino, promovendo a interface entre as diversas profissões e convergindo para uma atuação interdisciplinar, mas esse é ainda um desafio presente na formação em saúde, pois preceptores relatam falta de conhecimento e familiaridade com o papel, o que impactaria suas habilidades como preceptores (Fonseca et al., 2020).

O estudo de Ferreira e Souza (2019) refere estudos que apontam a IES como responsável por assumir a formação dos preceptores, através de práticas colaborativas e capacitação que estimule o desenvolvimento de aprendizagem problematizadora com a utilização de metodologias ativas de ensino/aprendizagem.

No entanto, é necessário refletir que a formação também do preceptor pelas IES é um grande desafio, considerando a demanda de atividades desenvolvidas por essas instituições (Fonseca et al., 2020).

Os resultados corroboraram a necessidade de mudanças no sentido de possibilitar aos alunos uma formação que os prepare melhor para uma atuação profissional mais humanizada, onde o trabalho em equipe e as práticas colaborativas sejam enfocadas nos conteúdos teóricos e práticos.

\section{Conclusão}

A presente pesquisa levantou um tema fundamental no processo de ensino-aprendizagem e que necessita ser explorado na graduação, nos programas de residência de enfermagem e também multiprofissionais, possibilitando uma reflexão baseada no conceito polissêmico e amplo que a humanização do cuidado em saúde preponde, articulado com as dificuldades cotidianas que os profissionais de saúde encontram na aplicabilidade deste conceito, baseado na PNH, principalmente no contexto da formação.

As interpretações produzidas a partir dos dados coletados demonstram que os preceptores possuem algum conhecimento acerca do tema, em concordância aos direcionamentos previstos pela PNH, porém de maneira dispersa. Tal percepção sugere certa incoerência entre o discurso do cuidado humanizado, preconizado pela PNH, e a prática assistencial, configurando a existência das práxis no cotidiano de trabalho. Atribui-se essa problemática à falta de uma reflexão mais ampla sobre a humanização em saúde, desde a formação profissional, buscando valorizar o processo de desenvolvimento de competências ético-morais que orientem a futura prática.

Entende-se, igualmente, a importância do constante estímulo ao educando, em seu ambiente de prática, como discussões relativas ao tema, tendo em vista o esclarecimento e sistematização dessas ações, além da promoção de debates e trocas de experiências sobre os processos que regem o cotidiano de todos envolvidos com os cuidados, no sentido de integrar a teoria e a prática. Nessa perspectiva, uma estratégia muito utilizada e que pode ter efeito positivo na equipe que foi estudada, são as rodas de conversa entre os profissionais, com encontros regulares que promovam o compartilhamento de experiências e saberes de cada profissional, bem como a resolução de conflitos. Além disso, as rodas de conversa levam a equipe a repensar sobre a atuação de cada membro, desencadeando iniciativas como o processo de humanização do cuidado.

As tecnologias de cuidado são recursos fundamentais na recuperação do paciente, exigem conhecimento e 
capacitação dos profissionais para que o seu manejo contribua na assistência. Porém, sem descaracterizar o cuidado pautado na compreensão subjetiva das reais necessidades do paciente, o que pode dificultar a aplicação e o exercício diário do cuidado humano integral. De tal modo, torna-se necessário a busca por novas estratégias, capazes de envolver a integralidade do cuidado. Acredita-se ter atingindo o objetivo deste estudo, contudo, é reconhecida a necessidade de maior investimento no período de formação dos profissionais.

Outrossim, estudos futuros podem ser realizados para se conhecer a realidade da humanização do cuidado na atuação de preceptores de outras instituições de saúde tanto a nível hospitalar, quanto em outros cenários, como na Atenção Básica, assim como outras tecnologias educacionais podem ser elaboradas com vistas a melhorar a qualidade da atenção à saúde prestada por este público.

\section{Referências}

Avila, L. I. et al. (2018). Construção moral do estudante de graduação em enfermagem como fomento da humanização do cuidado. Texto Contexto Enferm. 27(3), 1-9.

Bresolin, P. et al. (2019). Aprendizagem experiencial e diretrizes curriculares nacionais de enfermagem: revisão integrativa de literatura. Cogitare Enfermagem, 24. e59024.

Bonin, J. A. (2018). Processos e percursos de construção de pesquisas em recepção: algumas reflexões epistêmico-metodológicas. Conexão - Comunicação e Cultura, UCS, 17. Dossiê, 13-25. http://www.ucs.com.br/etc/revistas/index.php/conexao/article/view/6568

Costa, S. T. L. C. (2017). O preceptor na Residência em Terapia Intensiva: Competência na formação do enfermeiro. 106. Dissertação (Mestrado Profissional) - Universidade Federal de São Paulo. Programa de Pós-Graduação em Ensino em Ciências da Saúde.

Cox, C. D. et al. (2017). Use of entertainment elements in an online video mini-series to train pharmacy preceptors. American Journal of Pharmaceutical Education. 81(1). 1-9. https://doi.org/10.24926/iip.v8i2.522

Esperón, J. M. T. (2017). Pesquisa quantitativa na ciência da enfermagem. Escola Anna Nery, 21(1). 1-2.

Ferreira, B. J., \& Souza, S. V. (2019). Preceptoria: perspectivas e desafios na residência multiprofissional em saúde. ABCS Health Sciences, 44(1), 15-21. https://dx.doi.org/10.7322/abcshs.v44i1.1074

Freitas, F. D. S. \& Ferreira, M. A. (2016). Humanization knowledge of undergraduate nursing students. Rev Bras Enferm. 69(2), 261-8.

Fonseca, J. P. et al. (2020). Formação de preceptores na residência Multiprofissional ou Uniprofissional na área da saúde: uma revisão in tegrativa. Research, Society and Development, 9(8), e699986299-e699986299.

Luiz, F. F., Caregnato, R. C. A. \& Costa, M. R. (2017). Humanização na Terapia Intensiva: percepção do familiar e do profissional de saúde. Revista Brasileira de Enfermagem, 70(5), 1040-1047.

Monteiro, R. L. S. G. \& Santos, D. S. (2019). A utilização a ferramenta Google Forms como instrumento de avaliação do ensino na Escola Superior de Guerra. Revista Carioca de Ciência, Tecnologia e Educação. 4(2), 27-38.

Pereira, A. S. et al. (2018). Metodologia da pesquisa científica. UFSM. 1-119. https://repositorio.ufsm.br/bitstream/handle/1/15824/Lic_ Computacao_Metodologia-Pesquisa-Cientifica.pdf.

Prestes, M. L. M. (2012). A Pesquisa e a Construção do Conhecimento Científico: do planejamento aos textos, da escola a academia. (4a ed.), Rêspel.

Oliveira, B. M. F., \& Daher, D. V. (2016). A prática educativa do enfermeiro preceptor no processo de formação: o ensinar e o cuidar como participantes do mesmo processo. Rev. Docência Ens. Sup. 6(1), 113-138.

Rodrigues, A. M. et al. (2014). Preceptorship in the perspective of comprehensive care: conversations with nurses. Rev. Gaúcha Enferm. 35(2), 106-112.

Sanches, R. C. N. et al. (2016). Percepções de profissionais de saúde sobre a humanização em unidade de terapia inten siva adulto. Escola Anna Nery Revista de Enfermagem. 20(1), 48-54.

Santino, C. N. (2018). Metodologia para mapeamento das perdas em um processo de fundição, com aplicação da Escala Likert e da Lógica fuzzy. 1-116. Repositório UFBA.

Shimazu, T. (2018). The development of a public health nurses precepting experiential learning scale. Japanese journal of public health, 65(8), 377-85. https://doi.org/10.11236/jph.65.8_377 